\title{
Forecasting Human-Object Interaction: Joint Prediction of Motor Attention and Actions in First Person Video
}

\author{
Miao Liu ${ }^{1}$, Siyu Tang ${ }^{3}$, Yin $\mathrm{Li}^{2}$, and James M. Rehg ${ }^{1}$ \\ 1 Georgia Institute of Technology, Atlanta, United States \\ 2 University of Wisconsin-Madison, Madison, United States \\ 3 ETH Zürich, Switzerland
}

\begin{abstract}
We address the challenging task of anticipating human-object interaction in first person videos. Most existing methods either ignore how the camera wearer interacts with objects, or simply considers body motion as a separate modality. In contrast, we observe that the intentional hand movement reveals critical information about the future activity. Motivated by this observation, we adopt intentional hand movement as a feature representation, and propose a novel deep network that jointly models and predicts the egocentric hand motion, interaction hotspots and future action. Specifically, we consider the future hand motion as the motor attention, and model this attention using probabilistic variables in our deep model. The predicted motor attention is further used to select the discriminative spatial-temporal visual features for predicting actions and interaction hotspots. We present extensive experiments demonstrating the benefit of the proposed joint model. Importantly, our model produces new state-of-the-art results for action anticipation on both EGTEA Gaze+ and the EPIC-Kitchens datasets. Our project page is available at https://aptx4869lm.github.io/ForecastingHOI/
\end{abstract}

Keywords: First Person Vision, Action Anticipation, Motor Attention

\section{Introduction}

The human ability of "looking into the near future" remains a key challenge for computer vision. Consider the example in Fig. 1, given a video shortly before the start of an action, we can easily predict what will happen next, e.g., the person will take the canister of salt. Even without seeing any future frames, we can vividly imagine how the person will perform the action, e.g., the trajectory of the hand when reaching for the canister or the location on the canister that will be grasped.

There is convincing evidence that our remarkable ability to forecast other individuals' actions depends critically upon our perception and interpretation of their body motion. The investigation of this anticipatory mechanism dates back to 19th century, when William James argued that future expectations are intrinsically related to purposive body movements [26]. Additional evidence for 


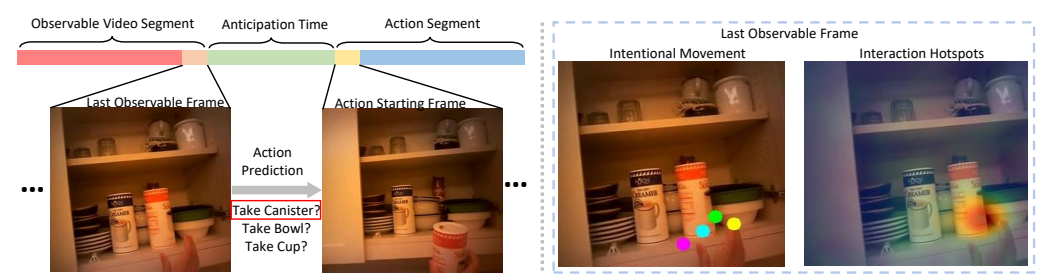

Fig. 1. What is the most likely future interaction? Our model takes advantage of the connection between motor attention and visual perception. In addition to future action label, our model also predicts the interaction hotspots on the last observable frame and hand trajectory (in the order of yellow, green, cyan, and magenta) between the last observable time step to action starting point. Visualizations of hand trajectory are projected to the last observable frame (best viewed in color).

a link between perceiving and performing actions was provided by the discovery of mirror neurons $[8,21]$. The observation of others' actions activates our motor cortex, the same brain regions that are in charge of the planning and control of intentional body motion. This activation can happen even before the onset of the action and is highly correlated with the anticipation accuracy [1]. A compelling explanation from [47] suggests that motor attention, i.e., the active prediction of meaningful future body movements, serves as a key representation for anticipation. A goal of this work is to develop a computational model for motor attention that can enable more accurate action prediction.

Despite these relevant findings in cognitive neuroscience, the role of intentional body motion in action anticipation is largely ignored by the existing literature $[58,11,16,28,13,15,39,29]$. In this work, we focus on the problem of forecasting human-object interactions in First Person Vision (FPV). Interactions consist of a single verb and one or more nouns, with "take bowl" as an example. FPV videos capture complex hand movements during a rich set of interactions, thus providing a powerful vehicle for studying the connection between motor attention and future representation. Several previous works have investigated the problems of FPV activity anticipation $[13,15]$ and body movement prediction $[2,20,12,59]$. We believe we are the first to utilize a motor attention model for FPV action anticipation.

To this end, we propose a novel deep model that predicts "motor attention"the future trajectory of the hands, as an anticipatory representation of actions. Based on motor attention, our model further localizes the future contact region of the interaction, i.e., interaction hotspots [41] and recognizes the type of future interactions. Importantly, we characterize motor attention and interaction hotspots as probabilistic variables modeled by stochastic units in a deep network. These units naturally deal with the uncertainty of future hand motion and contact region during interaction, and produce attention maps that highlight discriminative spatial-temporal features for action anticipation.

During inference, our model takes video clips shortly before the interaction as inputs, and jointly predicts motor attention, interaction hotspots, and action 
labels. During training, our model assumes that these outputs are available as supervisory signals. To evaluate our model, we report results on two major FPV benchmarks: EGTEA Gaze+ and EPIC-Kitchens. Our approach outperforms prior state-of-the-art methods by a significant margin. In addition, we conduct extensive ablation studies to verify the design of our model and evaluate our model for motor attention prediction and interaction hotspots estimation. Our model demonstrates strong results for both tasks. We believe our model provides a solid step towards the challenge of FPV visual anticipation.

\section{Related Works}

There has recently been substantial interest in learning to forecast future events in videos. The most relevant works to ours are those investigations on FPV action anticipation. Our work is also related to previous studies on third person action anticipation, other visual prediction tasks, and visual affordance.

FPV Action Anticipation. Action anticipation aims at predicting an action before it happens. We refer the readers to a recent survey [31] for a distinction between action recognition and anticipation. FPV action recognition has been studied extensively $[48,44,10,65,37,35,33,43]$, while fewer works have targeted egocentric action anticipation. Shen et al. [51] investigated how different egocentric modalities affect the action anticipation performance. Soran et al. [54] adopted Hidden Markov Model to compute the transition probability among sequences of actions. A similar idea was explored in [39]. Furnari et al. [13] considered the task of predicting the next-active objects. Their recent work [15] proposed to factorize the anticipation model into a "Rolling" LSTM that summarizes the past activity and an "Unrolling" LSTM that makes hypotheses of the future activity. Ke et al. [29] proposed a time-conditioned skip connection operation to extract relevant information for action anticipation. In contrast to our proposed method, these prior works did not exploit the connection between human motor attention and visual perception, and did not explicitly model the contact region during human-object interaction.

Third Person Action Anticipation. Several previous efforts seek to address the task of action anticipation in third person vision. Kris et al. [30] combined semantic scene labeling with a Markov decision process to forecast the behavior and trajectory of a subject. Vondrick et al. [58] proposed to predict the future video representation from large scale unlabeled video data. Gao et al. [16] proposed a Reinforced Encoder-Decoder network to create a summary representation of past frames and produce a hypothesis of future action. Kataoka et al. [28] introduced a subtle motion descriptor to identify the difference between an on-going action and a transitional action, and thereby facilitate future anticipation. Our work shares the same goal of future forecasting, but we focus on leveraging abundant visual cues from egocentric videos for action anticipation. Other Prediction Tasks. Anticipation has been studied under other vision tasks. In particular, human body motion prediction has been extensively studied $[42,57,60,20,59,12]$, including recent work in the setting of FPV. Rhinehart et 
al. [46] proposed an online learning algorithm to forecast the first-person trajectory. Park et al. [53] proposed a deep network to infer possible human trajectories from egocentric stereo images. Wei et al. [63] utilized a probabilistic model to infer 3D human attention and intention. Tagi et al. [64] addressed a novel task of predicting the future locations of an observed subject in egocentric videos. Ryoo et al. [49] proposed a novel method to summarize pre-activity observations for robot-centric activity prediction. However, none of these previous work considered modeling body movement for action anticipation.

Visual Affordance. The problem of predicting visual affordances has attracted growing interest in computer vision. Affordance can be helpful for scene understanding [18,7,61], human-object interaction recognition [55], and action analysis $[45,32]$. Several recent works have focused on estimating visual affordances that are grounded on human object interaction. Chen et al. [5] proposed to estimate likely object interaction regions by learning the connection between subject and object. Fang et al. [9] proposed to estimate interaction regions by learning from demonstration videos. However, none of these previous works considered future prediction. More recently, Tushar et al. [41] introduced an unsupervised learning method that uses the backward attention map to approximate the interaction hotspots grounded on a future action. However, their method did not model the presence of objects and thus can not be used to anticipate humanobject interactions. However, we compare to their results for interaction hotspot estimation in our experiments.

\section{Method}

We consider the setting of action anticipation from [6]. Denote an input video segment as $x:\left[\tau_{a}-\Delta \tau_{o}, \tau_{a}\right] . x$ starts at $\tau_{a}-\Delta \tau_{o}$ and ends at $\tau_{a}$ with duration $\Delta \tau_{o}>0$ as the "observation time". Our goal is to predict the label $y$ of an immediate future interaction starting at $\tau_{s}=\tau_{a}+\Delta \tau_{a}$, where $\Delta \tau_{a}>0$ is a fixed interval known as the "anticipation time." Moreover, we seek to estimate future hand trajectories $\mathcal{M}$ within $\left[\tau_{a}, \tau_{s}\right]$ (projected back to the last observable frame at $\tau_{a}$ ), and to localize interaction hotspots $\mathcal{A}$ at $\tau_{a}$ (the last observable frame). Fig. 1 illustrates our setting.

To summarize, our model seeks to anticipate the future action $y$ by jointly predicting the future hand trajectory $\mathcal{M}$ and interaction hotspots $\mathcal{A}$ at the last observable frame. Predicting the future is fundamentally ambiguous, since the observation of future interaction only represents one of the many possibilities characterized by an underlying distribution. Our key idea is thus to model motor attention and interaction hotspots as probabilistic variables in order to account for their uncertainty. We present an overview of our model in Fig. 2.

Specifically, we make use of a 3D backbone network $\phi(x)$ for video representation learning. Following the approach in $[52,22]$, we utilize 5 convolutional blocks, and denote the features from the $i^{t h}$ convolution block as $\phi_{i}(x)$. Based on $\phi(x)$, our motor attention module (b) predicts future hand trajectories as motor attention $\mathcal{M}$ and uses stochastic units to sample from $\mathcal{M}$. The sampled motor 


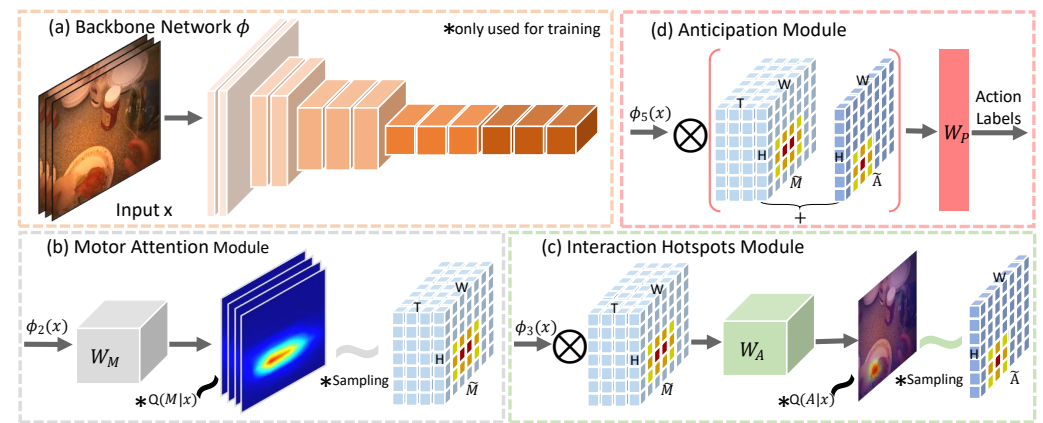

Fig. 2. Overview of our model. A 3D convolutional network $\phi(x)$ is used as our backbone network, with features from its $i^{\text {th }}$ convolution block as $\phi_{i}(x)$ (a). A motor attention module (b) makes use of stochastic units to generate sampled future hand trajectories $\tilde{\mathcal{M}}$ used to guide interaction hotspots estimation in module (c). Module (c) further generates sampled interaction hotspots $\tilde{\mathcal{A}}$ with a similar stochastic units as in module (b). Both $\tilde{\mathcal{M}}$ and $\tilde{\mathcal{A}}$ are used to guide action anticipation in anticipation module (d). During testing, our model takes only video clips as inputs, and predicts motor attention, interaction hotspots, and action labels. Note that $\otimes$ represents element-wise multiplication for weighted pooling.

attention $\tilde{\mathcal{M}}$ is an indicator of important spatial-temporal features for interaction hotspot estimation. Our interaction hotspot module (c) further produces an interaction hotspot distribution $\mathcal{A}$ and its sample $\tilde{\mathcal{A}}$. Finally, our anticipation module (d) makes use of both $\tilde{\mathcal{M}}$ and $\tilde{\mathcal{A}}$ to aggregate network features, and predicts the future interaction $y$.

\subsection{Joint Modeling of Human-Object Interaction}

Formally, we consider motor attention $\mathcal{M}$ and interaction hotspots $\mathcal{A}$ as probabilistic variables, and model the conditional probability of the future action label $y$ given the input video $x$ as a latent variable model, where

$$
p(y \mid x)=\int_{\mathcal{M}} \int_{\mathcal{A}} p(y \mid \mathcal{A}, \mathcal{M}, x) p(\mathcal{A} \mid \mathcal{M}, x) p(\mathcal{M} \mid x) d \mathcal{A} d \mathcal{M},
$$

$p(\mathcal{M} \mid x)$ first estimates motor attention from video input $x . \mathcal{M}$ is further used to estimate interaction hotspots $A(p(\mathcal{A} \mid \mathcal{M}, x))$. Given $x, \mathcal{M}$ and $\mathcal{A}$, the action label $y$ is determined by $p(y \mid \mathcal{A}, \mathcal{M}, x)$. Our model thus consists of three main components.

Motor Attention Module tackles $p(\mathcal{M} \mid x)$. Given the network features $\phi_{2}(x)$, our model uses a function $F_{M}$ to predict motor attention $\mathcal{M}$. $\mathcal{M}$ is represented as a $3 \mathrm{D}$ tensor of size $T_{m} \times H_{m} \times W_{m}$. Moreover, $\mathcal{M}$ is normalized within each temporal slice, i.e., $\sum_{w, h} \mathcal{M}(t, w, h)=1$.

Interaction Hotspots Module targets at $p(\mathcal{A} \mid \mathcal{M}, x)$. Our model uses a function $F_{A}$ to estimate the interaction hotspots $\mathcal{A}$ based on the network feature 
$\phi_{3}(x)$ and sampled motor attention $\tilde{\mathcal{M}}$. $\mathcal{A}$ is represented as a $2 \mathrm{D}$ attention map of size $H_{a} \times W_{a}$. A further normalization constrained that $\sum_{w, h} \mathcal{A}(w, h)=1$. Anticipation Module makes use of the predicted motor attention and interaction hotspots for action anticipation. Specifically, sampled motor attention $\tilde{\mathcal{M}}$ and sampled interaction hotspots $\tilde{\mathcal{A}}$ are used to aggregate feature $\phi_{5}(x)$ via weighted pooling. An action anticipation function $F_{P}$ further maps the aggregated features to future action label $y$.

\subsection{Motor Attention Module}

Motor Attention Generation. The motor attention prediction function $F_{M}$ is composed of a linear function with parameter $W_{M}$ on top of network features $\phi_{2}(x)$. The linear function is realized by a $3 \mathrm{D}$ convolution and a softmax function is used to normalized the attention map. This is given by $\psi=$ $\operatorname{softmax}\left(W_{M}^{T} \phi_{2}(x)\right)$, where the output $\psi$ is a $3 \mathrm{D}$ tensor of size $T_{m} \times H_{m} \times W_{m}$. We further model $p(\mathcal{M} \mid x)$ by normalizing $\psi$ within each temporal slice:

$$
\mathcal{M}_{m, n, t}=\frac{\psi_{m, n, t}}{\sum_{m, n} \psi_{m, n, t}},
$$

where $\psi_{m, n, t}$ is the value at location $(m, n)$ and time step $t$ in the 3D tensor of $\psi$. And $\mathcal{M}$ can be considered as the expectation of $p(\mathcal{M} \mid x)$.

Stochastic Modeling. Modeling motor attention in the context of forecasting human-object interaction requires a mechanism for addressing the stochastic nature of motor attention in developing the joint model. Here, we propose to use stochastic units to model the uncertainty. The key idea is to sample from the motor attention distribution. We follow the Gumbel-Softmax and reparameterization trick introduced in $[27,38]$ to design a differentiable sampling mechanism:

$$
\tilde{\mathcal{M}}_{m, n, t} \sim \frac{\exp \left(\left(\log \psi_{m, n, t}+G_{m, n, t}\right) / \theta\right)}{\sum_{m, n} \exp \left(\left(\log \psi_{m, n, t}+G_{m, n, t}\right) / \theta\right)},
$$

where $G$ is a Gumbel Distribution used to sample from discrete distribution. This Gumbel-Softmax trick produces a "soft" sampling step that allows the direct back-propagation of gradients to $\psi \cdot \theta$ is the temperature parameter that controls the "sharpness" of the distribution. We set $\theta=2$ for all of our experiments.

\subsection{Interaction Hotspots Module}

The predicted motor attention $\mathcal{M}$ is further used to guide interaction hotspots estimation $p(\mathcal{A} \mid x)$ by considering the conditional probability

$$
p(\mathcal{A} \mid x)=\int_{\mathcal{M}} p(\mathcal{A} \mid \mathcal{M}, x) p(\mathcal{M} \mid x) d \mathcal{M} .
$$

In practice, $p(\mathcal{A} \mid x)$ is estimated using sampled motor attention $\tilde{\mathcal{M}}$ based on $p(\mathcal{A} \mid \tilde{\mathcal{M}}, x)$ and $p(\tilde{\mathcal{M}} \mid x)$. For each sample $\tilde{\mathcal{M}}, p(\mathcal{A} \mid \tilde{\mathcal{M}}, x)$ is defined by the interaction hotspots estimation function $F_{A} . F_{A}$ takes the input of a motor attention 
map $\tilde{\mathcal{M}}$ and $\phi_{3}(x)$, and has the form of a linear $2 \mathrm{D}$ convolution parameterzied by $W_{A}$ followed by a softmax function.

$$
p(\mathcal{A} \mid \tilde{\mathcal{M}}, x)=\operatorname{softmax}\left(W_{A}^{T}\left(\tilde{\mathcal{M}} \otimes \phi_{3}(x)\right)\right),
$$

where $\otimes$ is the Hadamard product (element-wise multiplication). The result $p(\mathcal{A} \mid \mathcal{M}, x)$ is a 2D map of size $H_{a} \times W_{a}$. Intuitively, $\tilde{\mathcal{M}}$ presents a spatialtemporal saliency map to highlight feature representation $\phi_{3}(x) . F_{A}$ thus normalizes (using softmax) the output of a linear model on the selected features $\tilde{\mathcal{M}} \otimes \phi_{3}(x)$, and is a convex function. Finally, a similar sampling mechanism as in Eq. 3 can be used to sample $\tilde{\mathcal{A}}$ from $p(\mathcal{A} \mid x)$.

\subsection{Anticipation Module}

We now present the last piece of our model - the action anticipation module. The action anticipation function $p(y \mid \mathcal{A}, \mathcal{M}, x)=F_{P}(\mathcal{A}, \mathcal{M}, x)$ is defined as a function of the sampled motor attention map (3D) $\tilde{\mathcal{M}}$, sampled interaction heatmap (2D) $\tilde{\mathcal{A}}$ and the network feature $\phi_{5}(x)$. This is given by

$$
p(y \mid \tilde{\mathcal{A}}, \tilde{\mathcal{M}}, x)=\operatorname{softmax}\left(W_{P}^{T} \Sigma\left(\tilde{\mathcal{M}} \otimes \phi_{5}(x)\right)+W_{P}^{T} \Sigma\left(\tilde{\mathcal{A}} \odot \phi_{5}(x)\right)\right),
$$

where $\otimes$ is again the Hadamard product. $\Sigma$ is the global average pooling operation that pools a vector representation from a $2 \mathrm{D}$ or $3 \mathrm{D}$ feature map. $\odot$ is to use a $2 \mathrm{D}$ map $(\tilde{\mathcal{A}})$ to conduct Hadamard product to the last temporal slice of a $3 \mathrm{D}$ tensor $\phi_{5}(x)$. This is because the interaction hotspots $\tilde{\mathcal{A}}$ is only defined on the last observable frame. $W_{P}$ is a linear function that maps the features into prediction logits. $F_{P}$ is a combination of linear operations followed by a softmax function, and thus remains a convex function.

\subsection{Training and Inference}

Training our proposed joint model is challenging, as $p(\mathcal{M} \mid x)$ and $p(\mathcal{A} \mid \mathcal{M}, x)$ are intractable. Fortunately, variational inference comes to the rescue.

Prior Distribution. During training, we assume that reference distributions of future hand position $Q(\mathcal{M} \mid x)$ and interaction hotspots $Q(\mathcal{A} \mid x)$ are known in prior. These distributions can be derived from manual annotation of $2 \mathrm{D}$ fingertips and interaction hotspots, as we will describe in Sec 4.1. A 2D isotropic Gaussian is further applied to the annotated 2D points, leading to the distributions of $Q(\mathcal{M} \mid x)$ and $Q(\mathcal{A} \mid x)$. If annotations are not available, we adopt uniform distributions for both $Q(\mathcal{M} \mid x)$ and $Q(\mathcal{A} \mid x)$.

Variational Learning. Our proposed model seeks to jointly predict motor attention $\mathcal{M}$, interaction hotspots $\mathcal{A}$, and the action label $y$. Therefore, we inject the posterior $p(\mathcal{A}, \mathcal{M} \mid x)$ into $p(y \mid x)$. We further assume $p(\mathcal{A}, \mathcal{M} \mid x)$ can be factorized into $p(\mathcal{A} \mid x)$ and $p(\mathcal{M} \mid x)$ (see supplementary materials for details). Our model thereby optimizes the resulting latent variable model by maximizing the 
Evidence Lower Bound (ELBO), given by ${ }^{4}$

$$
\begin{aligned}
\log p(y \mid x) & \left.\geq E_{p(\mathcal{A}, \mathcal{M} \mid x)}[\log p(y \mid \mathcal{A}, \mathcal{M}, x)]-\log (p(\mathcal{A}, \mathcal{M} \mid x))\right] \\
& =\sum_{\mathcal{A}, \mathcal{M}} \log p(y \mid \mathcal{A}, \mathcal{M}, x)-K L[p(\mathcal{A} \mid x) \| Q(\mathcal{A} \mid x)]-K L[p(\mathcal{M} \mid x) \| Q(\mathcal{M} \mid x)]
\end{aligned}
$$

Therefore, the loss function $\mathcal{L}$ is given by

$$
\mathcal{L}=-\sum_{\mathcal{A}, \mathcal{M}} \log p(y \mid \mathcal{A}, \mathcal{M}, x)+K L[p(\mathcal{A} \mid x) \| Q(\mathcal{A} \mid x)]+K L[p(\mathcal{M} \mid x) \| Q(\mathcal{M} \mid x)] .
$$

The first term in the loss function is the cross entropy loss for action anticipation. The last two terms use KL-Divergence to align the predicted distributions of motor attention $p(\mathcal{M} \mid x)$ and interaction hotspots $p(\mathcal{A} \mid x)$ to their reference distributions $(Q(\mathcal{M} \mid x)$ and $Q(\mathcal{A} \mid x))$. To make the training practical, we draw a single sample for each input within a mini-batch similar to [27,38]. Multiple samples of the same input will be drawn at different iterations.

Approximate Inference. At inference time, our model could have drawn many samples of motor attention $\tilde{\mathcal{M}}$ and interaction hotspots $\tilde{\mathcal{A}}$ for the anticipation. However, the sampling and averaging is computationally expensive. We choose to feed deterministic $\mathcal{M}$ and $\mathcal{A}$ into Eq. 5 and Eq. 6 at inference time. Note that $F_{A}$ and $F_{P}$ are convex, since they are composed of linear mapping function and softmax function. By Jensen's inequality, we have

$$
\begin{gathered}
E\left[F_{A}(\tilde{\mathcal{M}}, x)\right] \geq F_{A}(E[\tilde{\mathcal{M}}], x)=F_{A}(\mathcal{M}, x), \\
E\left[F_{P}(\tilde{\mathcal{A}}, \tilde{\mathcal{M}}, x)\right] \geq F_{P}(E[\tilde{\mathcal{A}}], E[\tilde{\mathcal{M}}], x)=F_{P}(\mathcal{A}, \mathcal{M}, x)
\end{gathered}
$$

Therefore, such approximation provides a valid lower bound of $E\left[F_{P}(\tilde{\mathcal{A}}, \tilde{\mathcal{M}}, x)\right]$ and $E\left[F_{A}(\tilde{\mathcal{M}}, x)\right]$, and serves as a shortcut to avoid sampling during testing.

\subsection{Network Architecture}

We consider two different backbone networks for our model, including lightweight I3D-Res50 network [4,62] pre-trained on Kinetics and heavy CSN-152 [56] network pre-trained on IG-65M [17]. We use I3D-Res50 for our ablation study on EGTEA and EPIC-Kitchens, and report results using CSN-152 backbone when competing on the EPIC-Kitchens dataset. Both networks have five convolutional blocks. The motor attention module, the interaction hotspots module and the recognition module are attached to the 2 nd, the $3 \mathrm{rd}$ and the 5 th block, respectively. We use 3D max pooling to match the size of attention map to the size of the feature map in Eq. 5 and Eq. 6. For training, our model takes an input of 32 frames (every other frame from a 64 -frame chunk) with a resolution of $224 \times 224$. For inference, our model samples 30 clips from a video (3 along width of frame and 10 in time). Each clip has 32 frames with a resolution of $256 \times 256$. We average the scores of all sampled clips for video level prediction. Other implementation details will be discussed in the experiments.

\footnotetext{
${ }^{4}$ See supplementary material for the derivation.
} 


\section{Experiments}

We now present our experiments and results. We briefly introduce our implementation details and describe the datasets and annotations. Moreover, we present our results on EPIC-Kitchens action anticipation challenge, followed by ablation studies that further evaluate our model on interaction hotspot estimation and motor attention prediction. Finally, we provide a discussion of our method.

Implementation Details. Our model is trained using SGD with momentum 0.9 and batch size 64 on 4 GPUs. The initial learning rate is $2.5 \mathrm{e}-4$ with cosine decay. We set weight decay to 1e-4 and enable batch norm [25]. We downsample all frames to $320 \times 256$ (24fps) for EGTEA, and 512x288 (30fps) for EPICKitchens. We apply several data augmentation techniques, including random flipping, rotation, cropping and color jittering to avoid overfitting.

\subsection{Datasets and Annotations}

Datasets. We make use of two FPV datasets: EGTEA Gaze+ [33,34] and Epic-Kitchens [6]. EGTEA comes with 10,321 action instances from 19/53/106 verb/noun/action classes. We report results on the first split of the dataset. EPIC-Kitchens contains 39, 596 instances from 125 verbs and 352 nouns. We follow [15] to split the public training set into training and validation sets with 2513 action classes. We conduct ablation studies on this train/val split, and present the action anticipation results on the testing sets. We set the anticipation time as 0.5 seconds for EGTEA and 1 second [6] for EPIC-Kitchens.

Annotations. Our model requires supervisory signals of interaction hotspots and hand trajectories during training. We provide extra annotations for both EGTEA and EPIC-Kitchens datasets. These annotations will be made publicly available. Specifically, we manually annotated interaction hotspots as 2D points on the last observable frames for all instances on EGTEA and a subset of instances on EPIC-Kitchens. This is because many noun labels in EpicKitchens have very few instances, hence we focus on interaction hotspots of action instances that include many-shot nouns [6] in the training set.

Moreover, we explore different approaches to generate the pseudo ground truth of future hand trajectories. On EGTEA, we trained a hand segmentation model ([36] using hand masks from the dataset). The motor attention was approximated by segmenting hands at every frame and tracking the fingertip closest to an active object. To mitigate ego-motion, we used optical flow and RANSAC to compute a homography transform, and project the motor attention to the last observable frame. As EPIC-Kitchens does not provide hand masks, we instead annotated the fingertip closest to an interaction hotspots on the last observable frame. A linear interpolation of 2D motion between the fingertip and the interaction hotspots was used to approximate the motor attention.

\subsection{FPV Action Anticipation on EPIC-Kitchens}

We highlight our results for FPV action anticipation on EPIC-Kitchens dataset. 
Table 1. Action anticipation results on Epic-Kitchens. Ours+Obj model outperforms state-of-the-art by a notable margin. See discussions of Ours+Obj in Sec. 4.2.

\begin{tabular}{|c|c|c|c|c|}
\hline \multirow{2}{*}{\multicolumn{2}{|c|}{ Method }} & \multicolumn{3}{|c|}{ Top1/Top5 Accuracy } \\
\hline & & Verb & Noun & Action \\
\hline \multirow{7}{*}{ s1 } & 2SCNN [6] & $29.76 / 76.03$ & $15.15 / 38.65$ & $4.32 / 15.21$ \\
\hline & TSN [6] & $31.81 / 76.56$ & $16.22 / 42.15$ & $6.00 / 18.21$ \\
\hline & TSN+MCE [14] & $27.92 / 73.59$ & 16.09 / 39.32 & $10.76 / 25.28$ \\
\hline & Trans R(2+1)D [39] & $30.74 / 76.21$ & $16.47 / 42.72$ & $9.74 / 25.44$ \\
\hline & RULSTM [15] & $33.04 / \mathbf{7 9 . 5 5}$ & $22.78 / 50.95$ & $14.39 / 33.73$ \\
\hline & Ours & 34.99 / 77.05 & $20.86 / 46.45$ & $14.04 / 31.29$ \\
\hline & Ours + Obj & $\mathbf{3 6 . 2 5} / 79.15$ & $23.83 / 51.98$ & $15.42 / 34.29$ \\
\hline \multirow{7}{*}{$\mathrm{s} 2$} & 2SCNN [6] & $25.23 / 68.66$ & $9.97 / 27.38$ & $2.29 / 9.35$ \\
\hline & TSN [6] & $25.30 / 68.32$ & $10.41 / 29.50$ & $2.39 / 9.63$ \\
\hline & TSN+MCE [14] & $21.27 / 63.66$ & $9.90 / 25.50$ & $5.57 / 25.28$ \\
\hline & Trans $\mathrm{R}(2+1) \mathrm{D}$ [39] & $28.37 / 69.96$ & 12.43 / 32.20 & $7.24 / 19.29$ \\
\hline & RULSTM [15] & $27.01 / 69.55$ & 15.19 / 34.38 & $8.16 / 21.20$ \\
\hline & Ours & $28.27 / 70.67$ & $14.07 / 34.35$ & $8.64 / 22.91$ \\
\hline & Ours+Obj & $29.87 / 71.77$ & $16.80 / 38.96$ & $9.94 / 23.69$ \\
\hline
\end{tabular}

Experiment Setup. To compete for EPIC-Kitchens anticipation challenge, we used the backbone network CSN152. We trained our model on the public training set and report results using top-1/5 accuracy as in [6].

Results. Table 1 compares our results to latest methods on EPIC-Kitchens. Our model outperforms strong baselines (TSN and 2SCNN) reported in [6] by a large margin. Compared to previous best results from RULSTM [15], our model archives $+2 \% /-1.9 \% /-0.3 \%$ for verb/noun/action on seen set, and $+1.3 \% /-1.1 \% /+0.6 \%$ on unseen set of EPIC-Kitchens. Our results are better for verb, worse for noun and comparable or better for actions. Notably, RULSTM requires object boxes \& optical flow for training and object features \& optical flow for testing. In contrast, our method uses hand trajectories and interaction hotspots for training and needs only $R G B$ frames for testing.

To further improve the performance, we fuse the object stream from RULSTM with our model (Ours+Obj). Compared to RULSTM, Ours+Obj has a performance gain of $+3.2 \% /+2.9 \%$ for verb, $+1.1 \% /+1.6 \%$ for noun, and $+1.0 \% /+1.8 \%$ for action (seen/unseen). It is worthy pointing out that RULSTM benefits from an extra flow network, while ours+Obj model takes additional supervisory signals of hands and hotspots. Note that our performance boost does not simply come from those extra annotations. In a subsequent ablation study, we have shown that simply training with these extra annotations has minor improvement, when used without our proposed probabilistic deep model.

We note that it is not possible to make a direct apples-to-apples comparison between our model and RULSTM [15], as the two models used vastly different training signals. We refer readers to the supplementary materials for a detailed experiment setup comparison. In terms of performance, our model is comparable to RULSTM without using any side information for inference. When using addi- 
Table 2. Ablation study for action anticipation. We compare our model with backbone I3D network, and further analyze the role of motor attention prediction, interaction hotspots estimation, and stochastic units in joint modelling. See discussions in Sec. 4.3.

\begin{tabular}{c|ccc|ccc}
\multirow{2}{*}{ Method } & \multicolumn{4}{|c|}{ EGTEA } & \multicolumn{3}{c}{ Epic-Kitchens } \\
\cline { 2 - 7 } & \multicolumn{2}{|c|}{ Top1 Accuracy / Mean Cls Accuracy } & \multicolumn{2}{c}{ Top1 Accuracy / Top5 Accuracy } \\
& Verb & Noun & Action & Verb & Noun & Action \\
\hline I3D-Res50 & $48.01 / 31.25$ & $42.11 / 30.01$ & $34.82 / 23.20$ & $30.06 / 76.86$ & $16.07 / 41.67$ & $9.60 / 24.29$ \\
JointDet & $48.58 / 32.21$ & $43.95 / 31.26$ & $35.69 / 23.59$ & $30.16 / \mathbf{7 6 . 8 6}$ & $16.25 / 41.71$ & $9.76 / 24.40$ \\
Hotspots Only & $47.95 / 31.94$ & $44.02 / 32.53$ & $35.50 / 23.82$ & $30.21 / 75.93$ & $16.57 / 42.28$ & $9.66 / 24.33$ \\
Motor Only & $\mathbf{4 9 . 3 5 / 3 2 . 3 4}$ & $\mathbf{4 5 . 6 9} / \mathbf{3 3 . 9 3}$ & $36.49 / 25.13$ & $30.63 / 76.69$ & $17.28 / 42.56$ & $10.21 / 25.32$ \\
Ours & $48.96 / \mathbf{3 2 . 4 8}$ & $45.50 / 32.73$ & $\mathbf{3 6 . 6 0} / \mathbf{2 5 . 3 0}$ & $\mathbf{3 0 . 6 5} / 76.53$ & $\mathbf{1 7 . 4 0 / 4 2 . 6 0}$ & $\mathbf{1 0 . 3 8} / \mathbf{2 5 . 4 8}$
\end{tabular}

Table 3. Ablation study for interaction hotspots estimation. Jointly modeling motor attention with stochastic units can greatly benefit the performance of interaction hotspots estimation. ( $\uparrow / \downarrow$ indicates higher/lower is better) See discussions in Sec. 4.3.

\begin{tabular}{c|cccc|cccc} 
& \multicolumn{5}{|c|}{ EGTEA } & \multicolumn{4}{c}{ Epic-Kitchens } \\
\cline { 2 - 9 } Method & Prec $\uparrow$ & Recall $\uparrow$ & F1 $\uparrow$ & KLD $\downarrow$ & Prec $\uparrow$ & Recall $\uparrow$ & F1 $\uparrow$ & KLD $\downarrow$ \\
\hline I3DHeatmap & 12.82 & 37.53 & 19.11 & 2.66 & 17.20 & 77.39 & 28.15 & 3.07 \\
JointDet & 16.11 & 41.82 & 23.26 & 1.84 & 17.32 & 85.79 & 28.83 & 2.21 \\
Ours & $\mathbf{1 7 . 4 3}$ & $\mathbf{4 8 . 8 1}$ & $\mathbf{2 5 . 6 9}$ & $\mathbf{1 . 6 2}$ & $\mathbf{1 7 . 8 6}$ & $\mathbf{8 6 . 5 9}$ & $\mathbf{2 9 . 6 0}$ & $\mathbf{1 . 9 9}$
\end{tabular}

tional object stream during inference as in RULSTM, our model outperforms RULSTM by a relative improvement of $\mathbf{7 \%} / \mathbf{2 2 \%}$ on seen/unseen set. More importantly, our model also provides the additional capabilities of predicting future hand trajectories and estimating interaction hotspots.

\subsection{Ablation Study}

We present ablation studies of our model. We introduce our experiment setup, evaluate each component of our model, and then contrast our method to a series of baselines on motor attention prediction and interaction hotspot estimation

Experiment Setup. For all of our ablation studies, we adopt the lightweight I3D-Res50 [62] as backbone network to reduce computational cost. Our model is evaluated for action anticipation, motor attention prediction and interaction hotspots estimation across EGTEA (using split1) and EPIC-Kitchens (using the train/val split from [15]). Specifically, we consider the following metrics.

- Action Anticipation. We report Top1/Mean Class accuracy on EGTEA as in [35] and Top1/Top5 accuracy as on EPIC-Kitchens following [15].

- Interaction Hotspots Estimation. We report F1 score as in [33] and KLDivergence (KLD) as in [41] using a downsampled heatmap (32x) at the last observable frame.

- Motor Attention Prediction. We report the average and final displacement errors between the most confident location on a predicted attention map and the ground-truth hand points, similar to previous work on trajectory prediction [3]. Note that the motor attention maps is downsampled by a factor of $32 / 8$ in space/time. Hence, we report displacement errors normalized in spatial and temporal dimension. 
Benefits of Joint Modeling. As a starting point, we compare our model with a backbone I3D-Res50 model. We present the results of action anticipation in Table 2. In comparison to I3D-Res50, our model improves noun and action prediction by $+3.4 \% / 1.8 \%$ on EGTEA and $+1.3 \% / 0.8 \%$ on EPIC-Kitchens. Moreover, we show that our model improves the performance of interaction hotspots estimation. We consider the baseline I3D model that only estimates interaction region with interaction hotspots module as I3DHeatmap. As shown in Table 3, our model improves the F1 score by $6.6 \% / 1.5 \%$ on EGTEA/EPIC-Kitchens.

Stochastic Modeling vs. Deterministic Modeling. We further evaluate the benefits of probabilistic modeling of motor attention and interaction hotspots. To this end, we compare our model with a deterministic joint model (JointDet). JointDet has the same architecture as our model, except for the stochastic units. As shown in Table 2, JointDet slightly improve the I3D baseline for action anticipation $(+0.87 \%$ on EGTEA and $+0.16 \%$ on EPIC-Kitchens), yet lags behind our probabilistic model. Specifically, our model outperforms JointDet by $0.91 \%$ and $0.62 \%$ on EGTEA and EPIC-Kitchens. Moreover, in comparison to JointDet, our model has better performance for interaction hotspots estimation $(+2.4 \% /+0.8 \%$ in F1 scores on EGTEA/EPIC-Kitchens). These results suggest that simply training with extra annotations might fail to capture the uncertainty of visual anticipation. In contrast, our design choice of probabilistic modeling can effectively deal with those uncertainty, therefore helps to improve the performance of joint modeling.

Motor Attention vs. Interaction Hotspots. Futhermore, we evaluate the contributions of motor attention and interaction hotspots for FPV action anticipation. We consider two baseline models in Table 3: I3D model equipped with only motor attention module (Motor Only), and I3D model equipped with only interaction hotspots module (Hotspots Only). Both models underperform the full model across the two datasets, yet the gap between Motor Only and the full model is smaller. These results suggest that both components contribute to the performance boost of action anticipation, yet the modeling of motor attention weights more than the modeling of interaction hotspots.

Interaction Hotspots Estimation. We present additional results on interaction hotspots estimation. We compare our results to the following baselines.

- Center Prior represents a Gaussian Distribution at the center of the image.

- Grad-Cam uses the same I3D backbone network as our model, and produces a saliency map via Grad-Cam [50].

- EgoGaze considers possible gaze position as salient region of a given image. This model is trained on eye fixation annotation from EGTEA-Gaze+ [24]. The assumption is that the person is likely to look at the interaction hotspots.

- DSS Saliency predicts salient region during human object interaction. This model is trained on pixel-level saliency annotation from [23].

- EgoHotspots is the latest work [41] for estimating interaction hotspots.

Our results are shown in Table 4. Our model outperforms the best baselines (EgoGaze and EgoHotspots) by $5.4 \%$ on EGTEA and $3.6 \%$ on EPIC-Kitchens 
Table 4. Interaction hotspots estimation results on EGTEA and EPIC-Kitchens. Our model outperforms a set of strong baselines. ( $\uparrow / \downarrow$ indicates higher/lower is better)

\begin{tabular}{|c|c|c|c|c|c|c|c|c|}
\hline \multirow[b]{2}{*}{ Method } & \multicolumn{4}{|c|}{ EGTEA } & \multicolumn{4}{|c|}{ Epic-Kitchens } \\
\hline & Prec $\uparrow$ & Recall $\uparrow$ & $\mathrm{F} 1 \uparrow$ & KLD $\downarrow$ & Prec $\uparrow$ & Recall $\uparrow$ & $\mathrm{F} 1 \uparrow$ & KLD $\downarrow$ \\
\hline Center Prior & 10.87 & 17.65 & 13.45 & 10.64 & 11.66 & 16.97 & 13.82 & 10.27 \\
\hline Grad-Cam [50] & 9.98 & 22.13 & 13.76 & 8.73 & 10.85 & 20.01 & 14.07 & 8.06 \\
\hline DSS [23] & 9.02 & 39.49 & 14.69 & 6.12 & 12.03 & 33.75 & 17.74 & 5.21 \\
\hline EgoGaze [24] & 15.02 & 31.34 & 20.31 & 3.20 & 11.30 & 27.65 & 16.05 & 3.37 \\
\hline EgoHotspots [41] & 16.51 & 24.07 & 19.59 & 3.36 & 22.26 & 31.37 & 26.04 & 2.84 \\
\hline Ours & 17.43 & 48.81 & 25.69 & 1.62 & 17.86 & 86.5 & 29.6 & 1.99 \\
\hline
\end{tabular}

Table 5. Motor attention prediction results on EGTEA. Our model compares favourably to strong baselines. ( $\uparrow / \downarrow$ indicates higher/lower is better)

\begin{tabular}{c|c|c} 
Method & Avg. Disp. Error $\downarrow$ & Final Disp. Error $\downarrow$ \\
\hline Kalman Filter & 0.32 & 0.48 \\
GPR & 0.29 & 0.37 \\
LSTM & $\mathbf{0 . 2 2}$ & $\mathbf{0 . 3 5}$ \\
Ours & 0.23 & 0.36
\end{tabular}

in F1 scores. These results suggest that our proposed joint model can effectively identify future interaction region. Another observation is that our model performs better on EPIC-Kitchens than EGTEA. This is probably due to the larger number of available training samples.

Motor Attention Prediction. We report our results on motor attention prediction. We consider the following baselines and only report results on EGTEA, as the future hand position on EPIC-Kitchens is not accurate (see Sec. 4.1).

- Kalman Filter describes the hand trajectory prediction problem with statespace model, and assumes linear acceleration during update step.

- Gaussian Process Regression (GPR) iteratively predicts the future hand position using Gaussian Process Regression.

- LSTM adopts a vanilla LSTM network for trajectory forecasting. We use the implementation from [3].

The results are presented in Table 5. Our model outperforms Kalman filter and GPR, yet is slightly worse than LSTM model ( +0.01 in both errors). Note that all baseline methods need the coordinate of the first observed hand for prediction. This simplifies trajectory prediction into a less challenging regression problem. In contrast, our model does not need hand coordinates for inference. A model that relies on the observation of hand positions will encounter failure cases when the hand has not been observed, while our model is still capable of "imagining" the possible hand trajectory. See "Operate Microwave" and "Wash Coffee Cup" in Fig. 3 for example results from our model.

Visualization of Motor Attention and Interaction Hotspots. Finally, we visualize the predicted motor attention, interaction hotspots, and action labels from our model in Fig. 3. The predicted motor attention almost always attends to the predicted objects and corresponding interaction hotspots. Hence, our model can address challenging cases where next-active objects are ambiguous. Take the 

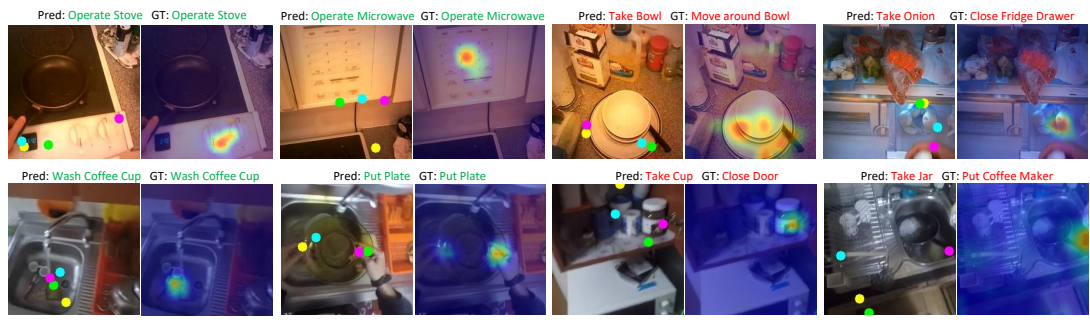

Fig. 3. Visualization of motor attention (left image), interaction hotspots (right image), and action labels (captions above the images) on sample frames from EGTEA (first row) and EPIC-Kitchens (second row). Both successful (green label) and failure cases (red label) are shown. Future hands position are predicted at every 8 frames and plotted on the last observable frame with the order of yellow, green, cyan, and magenta.

first example of "Operate Stove" in Fig. 3. Our model successfully predicted the future objects and estimated the interaction hotspots as the stove control knob.

\subsection{Remarks and Discussion}

We must also point out that our method has certain limitations, which point to exciting future research directions. For example, our model requires additional annotations for training, which might bring scalability issues when analyzing other datasets. These dense annotations can indeed be approximated using sparsely annotated frames as discussed in Sec. 4.1. We speculate that more advanced hand tracking and object segmentation models can be explored to generating the pseudo ground truth of motor attention and interaction hotspots. Moreover, our model shares a similar conundrum faced by previous work on anticipation. Our model is likely to fail when future active objects are not observed. See "Close Fridge Drawer" and "Put Coffee Maker" in Fig. 3. We conjecture that these cases requires incorporating logical reasoning into learning based methods - an active research topic in our community.

\section{Conclusions}

We presented the first deep model that jointly predicts motor attention, interaction hotspots, and future action labels in FPV. Importantly, we demonstrated that motor attention plays an important role in forecasting human-object interactions. Another key insight is that characterizing motor attention and interaction hotspots as probabilistic variables can account for the stochastic pattern of human intentional movement. We believe that our model provides a solid step towards the challenging problem of visual anticipation.

Acknowledgments. Portions of this research were supported in part by National Science Foundation Award 1936970 and a gift from Facebook. YL acknowledges the support from the Wisconsin Alumni Research Foundation. 


\section{References}

1. Aglioti, S.M., Cesari, P., Romani, M., Urgesi, C.: Action anticipation and motor resonance in elite basketball players. Nature neuroscience (2008)

2. Aksan, E., Kaufmann, M., Hilliges, O.: Structured prediction helps 3d human motion modelling. In: ICCV (2019)

3. Alahi, A., Goel, K., Ramanathan, V., Robicquet, A., Fei-Fei, L., Savarese, S.: Social LSTM: Human trajectory prediction in crowded spaces. In: CVPR (2016)

4. Carreira, J., Zisserman, A.: Quo vadis, action recognition? a new model and the kinetics dataset. In: CVPR (2017)

5. Chen, C.Y., Grauman, K.: Subjects and their objects: Localizing interactees for a person-centric view of importance. International Journal of Computer Vision 126(2-4), 292-313 (2018)

6. Damen, D., Doughty, H., Farinella, G.M., Fidler, S., Furnari, A., Kazakos, E., Moltisanti, D., Munro, J., Perrett, T., Price, W., Wray, M.: Scaling egocentric vision: The epic-kitchens dataset. In: Ferrari, V., Hebert, M., Sminchisescu, C., Weiss, Y. (eds.) ECCV. pp. 753-771. Springer International Publishing (2018)

7. Delaitre, V., Fouhey, D.F., Laptev, I., Sivic, J., Gupta, A., Efros, A.A.: Scene semantics from long-term observation of people. In: Fitzgibbon, A., Lazebnik, S., Perona, P., Sato, Y., Schmid, C. (eds.) ECCV. pp. 284-298. Springer Berlin Heidelberg (2012)

8. Di Pellegrino, G., Fadiga, L., Fogassi, L., Gallese, V., Rizzolatti, G.: Understanding motor events: a neurophysiological study. Experimental brain research (1992)

9. Fang, K., Wu, T.L., Yang, D., Savarese, S., Lim, J.J.: Demo2vec: Reasoning object affordances from online videos. In: CVPR (2018)

10. Fathi, A., Farhadi, A., Rehg, J.M.: Understanding egocentric activities. In: ICCV (2011)

11. Felsen, P., Agrawal, P., Malik, J.: What will happen next? forecasting player moves in sports videos. In: ICCV (2017)

12. Fragkiadaki, K., Levine, S., Felsen, P., Malik, J.: Recurrent network models for human dynamics. In: ICCV (2015)

13. Furnari, A., Battiato, S., Grauman, K., Farinella, G.M.: Next-active-object prediction from egocentric videos. VCIP (2017)

14. Furnari, A., Battiato, S., Maria Farinella, G.: Leveraging uncertainty to rethink loss functions and evaluation measures for egocentric action anticipation. In: LealTaixé, L., Roth, S. (eds.) ECCV Workshops. pp. 389-405. Springer International Publishing (2018)

15. Furnari, A., Farinella, G.M.: What would you expect? anticipating egocentric actions with rolling-unrolling LSTMs and modality attention. In: ICCV (2019)

16. Gao, J., Yang, Z., Nevatia, R.: Red: Reinforced encoder-decoder networks for action anticipation. In: BMVC (2017)

17. Ghadiyaram, D., Tran, D., Mahajan, D.: Large-scale weakly-supervised pretraining for video action recognition. In: CVPR (2019)

18. Grabner, H., Gall, J., Van Gool, L.: What makes a chair a chair? In: CVPR (2011)

19. Gu, C., Sun, C., Ross, D.A., Vondrick, C., Pantofaru, C., Li, Y., Vijayanarasimhan, S., Toderici, G., Ricco, S., Sukthankar, R., et al.: Ava: A video dataset of spatiotemporally localized atomic visual actions. In: CVPR (2018)

20. Gui, L.Y., Wang, Y.X., Liang, X., Moura, J.M.: Adversarial geometry-aware human motion prediction. In: Ferrari, V., Hebert, M., Sminchisescu, C., Weiss, Y. (eds.) ECCV. pp. 823-842. Springer International Publishing (2018) 
21. Hari, R., Forss, N., Avikainen, S., Kirveskari, E., Salenius, S., Rizzolatti, G.: Activation of human primary motor cortex during action observation: a neuromagnetic study. Proceedings of the National Academy of Sciences (1998)

22. He, K., Zhang, X., Ren, S., Sun, J.: Deep residual learning for image recognition. In: CVPR (2016)

23. Hou, Q., Cheng, M.M., Hu, X., Borji, A., Tu, Z., Torr, P.: Deeply supervised salient object detection with short connections. In: CVPR (2017)

24. Huang, Y., Cai, M., Li, Z., Sato, Y.: Predicting gaze in egocentric video by learning task-dependent attention transition. In: Ferrari, V., Hebert, M., Sminchisescu, C., Weiss, Y. (eds.) ECCV. pp. 789-804. Springer International Publishing (2018)

25. Ioffe, S., Szegedy, C.: Batch normalization: Accelerating deep network training by reducing internal covariate shift. In: ICML (2015)

26. James, W., Burkhardt, F., Bowers, F., Skrupskelis, I.K.: The principles of psychology, vol. 1. Macmillan London (1890)

27. Jang, E., Gu, S., Poole, B.: Categorical reparameterization with gumbel-softmax. In: ICLR (2017)

28. Kataoka, H., Miyashita, Y., Hayashi, M., Iwata, K., Satoh, Y.: Recognition of transitional action for short-term action prediction using discriminative temporal cnn feature. In: BMVC (2016)

29. Ke, Q., Fritz, M., Schiele, B.: Time-conditioned action anticipation in one shot. In: CVPR (2019)

30. Kitani, K.M., Ziebart, B.D., Bagnell, J.A., Hebert, M.: Activity forecasting. In: Fitzgibbon, A., Lazebnik, S., Perona, P., Sato, Y., Schmid, C. (eds.) ECCV. pp. 201-214. Springer Berlin Heidelberg (2012)

31. Kong, Y., Fu, Y.: Human action recognition and prediction: A survey. arXiv preprint arXiv:1806.11230 (2018)

32. Koppula, H.S., Saxena, A.: Anticipating human activities using object affordances for reactive robotic response. IEEE transactions on pattern analysis and machine intelligence 38(1), 14-29 (2015)

33. Li, Y., Liu, M., Rehg, J.M.: In the eye of beholder: Joint learning of gaze and actions in first person video. In: Ferrari, V., Hebert, M., Sminchisescu, C., Weiss, Y. (eds.) ECCV. pp. 639-655. Springer International Publishing (2018)

34. Li, Y., Liu, M., Rehg, J.M.: In the eye of the beholder: Gaze and actions in first person video. arXiv preprint arXiv:2006.00626 (2020)

35. Li, Y., Ye, Z., Rehg, J.M.: Delving into egocentric actions. In: CVPR (2015)

36. Long, J., Shelhamer, E., Darrell, T.: Fully convolutional networks for semantic segmentation. In: CVPR (2015)

37. Ma, M., Fan, H., Kitani, K.M.: Going deeper into first-person activity recognition. In: CVPR (2016)

38. Maddison, C.J., Mnih, A., Teh, Y.W.: The concrete distribution: A continuous relaxation of discrete random variables. In: ICLR (2017)

39. Miech, A., Laptev, I., Sivic, J., Wang, H., Torresani, L., Tran, D.: Leveraging the present to anticipate the future in videos. In: CVPR Workshops (2019)

40. Misra, I., Shrivastava, A., Gupta, A., Hebert, M.: Cross-stitch networks for multitask learning. In: CVPR (2016)

41. Nagarajan, T., Feichtenhofer, C., Grauman, K.: Grounded human-object interaction hotspots from video. In: ICCV (2019)

42. Pavlovic, V., Rehg, J.M., MacCormick, J.: Learning switching linear models of human motion. In: Leen, T.K., Dietterich, T.G., Tresp, V. (eds.) NeurIPS. pp. 981-987. MIT Press (2001) 
43. Pirsiavash, H., Ramanan, D.: Detecting activities of daily living in first-person camera views. In: CVPR (2012)

44. Poleg, Y., Ephrat, A., Peleg, S., Arora, C.: Compact CNN for indexing egocentric videos. In: WACV (2016)

45. Rhinehart, N., Kitani, K.M.: Learning action maps of large environments via firstperson vision. In: CVPR (2016)

46. Rhinehart, N., Kitani, K.M.: First-person activity forecasting with online inverse reinforcement learning. In: ICCV (2017)

47. Rushworth, M., Johansen-Berg, H., Göbel, S.M., Devlin, J.: The left parietal and premotor cortices: motor attention and selection. Neuroimage 20, S89-S100 (2003)

48. Ryoo, M.S., Rothrock, B., Matthies, L.: Pooled motion features for first-person videos. In: CVPR (2015)

49. Ryoo, M., Fuchs, T.J., Xia, L., Aggarwal, J.K., Matthies, L.: Robot-centric activity prediction from first-person videos: What will they do to me? In: HRI (2015)

50. Selvaraju, R.R., Cogswell, M., Das, A., Vedantam, R., Parikh, D., Batra, D.: Gradcam: Visual explanations from deep networks via gradient-based localization. In: ICCV (2017)

51. Shen, Y., Ni, B., Li, Z., Zhuang, N.: Egocentric activity prediction via event modulated attention. In: Ferrari, V., Hebert, M., Sminchisescu, C., Weiss, Y. (eds.) Proceedings of the European Conference on Computer Vision (ECCV). pp. 202217. Springer International Publishing (2018)

52. Simonyan, K., Zisserman, A.: Very deep convolutional networks for large-scale image recognition. In: ICLR (2015)

53. Soo Park, H., Hwang, J.J., Niu, Y., Shi, J.: Egocentric future localization. In: CVPR (2016)

54. Soran, B., Farhadi, A., Shapiro, L.: Generating notifications for missing actions: Don't forget to turn the lights off! In: ICCV (2015)

55. Thermos, S., Papadopoulos, G.T., Daras, P., Potamianos, G.: Deep affordancegrounded sensorimotor object recognition. In: CVPR (2017)

56. Tran, D., Wang, H., Torresani, L., Feiszli, M.: Video classification with channelseparated convolutional networks. In: ICCV (2019)

57. Urtasun, R., Fleet, D.J., Geiger, A., Popović, J., Darrell, T.J., Lawrence, N.D.: Topologically-constrained latent variable models. In: ICML (2008)

58. Vondrick, C., Pirsiavash, H., Torralba, A.: Anticipating visual representations from unlabeled video. In: CVPR (2016)

59. Walker, J., Marino, K., Gupta, A., Hebert, M.: The pose knows: Video forecasting by generating pose futures. In: ICCV (2017)

60. Wang, J.M., Fleet, D.J., Hertzmann, A.: Gaussian process dynamical models for human motion. IEEE transactions on pattern analysis and machine intelligence 30(2), 283-298 (2007)

61. Wang, X., Girdhar, R., Gupta, A.: Binge watching: Scaling affordance learning from sitcoms. In: CVPR (2017)

62. Wang, X., Girshick, R., Gupta, A., He, K.: Non-local neural networks. In: CVPR (2018)

63. Wei, P., Xie, D., Zheng, N., Zhu, S.C.: Inferring human attention by learning latent intentions. In: IJCAI (2017)

64. Yagi, T., Mangalam, K., Yonetani, R., Sato, Y.: Future person localization in firstperson videos. In: CVPR (2018)

65. Zhou, Y., Ni, B., Hong, R., Yang, X., Tian, Q.: Cascaded interactional targeting network for egocentric video analysis. In: CVPR (2016) 
This is the supplementary material for our submission to ECCV 2020, titled "Forecasting Human-Object Interaction: Joint Prediction of Motor Attention and Actions in First Person Video". The contents are organized as follows.

- A Network Architecture.

- B Mathematical Derivation for Equation 8.

- C Details on Data Annotation.

- D Experiment Setup Comparison to RULSTM.

- E Epic-Kitchens Challenge Leaderboard.

- F Experiments on Gaze Fixation Model.

- G Additional Qualitative Results.

\section{F Network Architecture}

Network Architecture. We present our network architecture using 3D Res50 backbone in Table 8. A similar architecture is also used for CSN-152 backbone. We followed [33] to use the features from bottom layers of the network for motor attention prediction and interaction hotspots estimation, and the features from the top layer for action anticipation. Our model jointly predicts motor attention, interaction hotspots and future actions, and thus is conceptually similar to multitask learning e.g., [40]. The key difference is that outputs of our model depends on each other. For example, motor attention is used for interaction hotspots estimation and both motor attention and interaction hotspots are used for action anticipation.

\section{G $\quad$ Mathematical Derivation for Equation 7}

We present the derivation of our variational learning as discussed in Sec 3.5. Specifically, we inject posterior $p(\mathcal{A}, \mathcal{M} \mid x)$ into $p(y \mid x)$ and optimize the resulting latent variable model by maximizing the Evidence Lower Bound (ELBO). However, the prior distribution of $Q(\mathcal{A}, \mathcal{M} \mid x)$ is not available for training. Hence, we further approximate $p(\mathcal{A}, \mathcal{M} \mid x)$ by factorizing it into $p(\mathcal{A} \mid x)$ and $p(\mathcal{M} \mid x)$. Namely, we assume that $\mathcal{A}$ and $\mathcal{M}$ is conditionally independent given the input $x$. Thus, we have

$$
\begin{aligned}
& K L[p(\mathcal{A}, \mathcal{M} \mid x) \| Q(\mathcal{A}, \mathcal{M} \mid x)] \\
= & K L[p(\mathcal{A} \mid \mathcal{M}, x) \| Q(\mathcal{A} \mid \mathcal{M}, x)]+K L[p(\mathcal{M} \mid x) \| Q(\mathcal{M} \mid x)] .
\end{aligned}
$$

The ELBO of our proposed joint model can be derived as

$$
\begin{aligned}
\log p(y \mid x) & \left.\geq E_{p(\mathcal{A}, \mathcal{M} \mid x)}[\log p(y \mid \mathcal{A}, \mathcal{M}, x)]-\log (p(\mathcal{A}, \mathcal{M} \mid x))\right] \\
& =\sum_{\mathcal{A}, \mathcal{M}} \log p(y \mid \mathcal{A}, \mathcal{M}, x)-K L[p(\mathcal{A}, \mathcal{M} \mid x) \| Q(\mathcal{A}, \mathcal{M} \mid x)] \\
& =\sum_{\mathcal{A}, \mathcal{M}} \log p(y \mid \mathcal{A}, \mathcal{M}, x)-K L[p(\mathcal{A} \mid x) \| Q(\mathcal{A} \mid x)]-K L[p(\mathcal{M} \mid x) \| Q(\mathcal{M} \mid x)]
\end{aligned}
$$




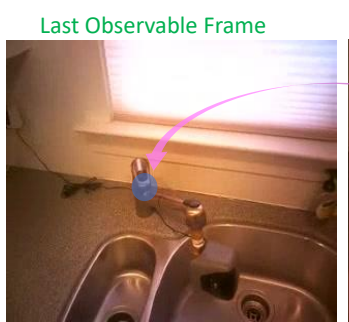

(a) Interaction Hotspots Annotation

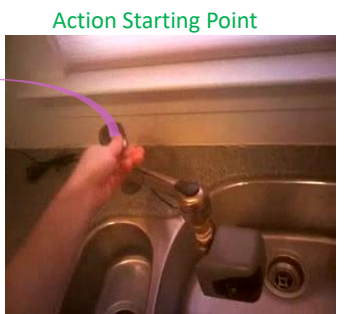

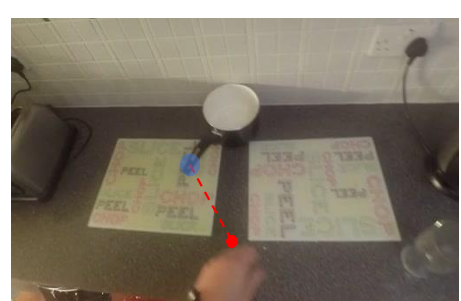

(b) Approximated Hand Trajectory

Fig. 4. (a) illustrates the interaction hotspots annotation process. (b) illustrates the approximation of the future hand trajectory on the Epic-Kitchens dataset.

Table 6. Comparison between our methods and previous state-of-the-art results RULSTM. See Sec.4.2 of our submission for discussion of Ous+Obj.

\begin{tabular}{ccccc} 
Method & Tasks & Training Supervision & Testing Inputs & End-to-End \\
\hline RULSTM [15] & Action Anticipation & $\begin{array}{c}\text { Action Labels } \\
\text { Object Cls \& Boxes }\end{array}$ & $\begin{array}{c}\text { RGB + Object Feat. } \\
\text { + Flow }\end{array}$ & No \\
\hline Ours & $\begin{array}{c}\text { Action Anticipation } \\
\text { Visual Affordance } \\
\text { Motor Attention Pred }\end{array}$ & $\begin{array}{c}\text { Action Labels } \\
\text { Hand \& Hotspots }\end{array}$ & RGB & Yes \\
\hline Ours+Obj & $\begin{array}{c}\text { Action Anticipation } \\
\text { Visual Affordance } \\
\text { Motor Attention Pred }\end{array}$ & $\begin{array}{c}\text { Action Labels } \\
\text { Object Cls \& Boxes } \\
\text { Hand \& Hotspots }\end{array}$ & RGB + Object Feat. & No
\end{tabular}

\section{H Details on Data Annotation}

We provide additional details on data annotation. In Sec. 4.1, we introduced how we obtain the prior distribution of motor attention and interaction htospots. Here we provide a visual illustration of our efforts on the data annotation. As shown in Fig. 4 (a), we compare the last observable frame with the first frame of action segment. If the active object presents in the last observable frame, we annotate the corresponding contact point and enforce a 2D Gaussian distribution to imitate the stochastic patterns of human-object interaction. Since the hand mask is absent from EPIC-Kitchens dataset, we adopt a 2D interpolation between the the finger tip annotation and interaction hotspots annotation to generate the pseudo ground truth of future hand trajectory (Take Fig. 4 (b) for an instance). Note that we use a smaller anticipation time $(0.5 \mathrm{~s})$ on the EGTEA dataset. This is because the EGTEA dataset has a smaller angle of view in comparison with the EPIC-Kitchens dataset. A large anticipation time will reduce the number of samples that have next-active objects on the last observable frame. To summarize, there are 14951 annotated sample on the EPIC-Kitchens Dataset, and 7381 annotated samples on the EGTEA dataset. We believe those additional annotations can facilitate future research of human-object interaction in FPV. 
Table 7. Additional results on fixation based model. We contrast Gaze Only Model with baseline I3D model and our full model.

\begin{tabular}{c|ccc} 
Methods & Verb & Noun & Action \\
\hline I3D-Res50 & $48.01 / 31.25$ & $42.11 / 30.01$ & $34.82 / 23.20$ \\
Gaze Only $^{\dagger}$ & $47.88 / 31.79$ & $43.83 / 33.42$ & $35.31 / 24.51$ \\
Ours $^{\dagger}$ & $48.96 / 32.48$ & $45.50 / 32.73$ & $36.60 / 25.30$
\end{tabular}

\section{Comparison of Experiment Setup to RULSTM}

We highlight our comparison to RULSTM. In Sec.4.2 of our submission, we contrast our method with previous state-of-the-art result RULSTM [15]. Here, we draw a more clear boundary between our method and RULSTM. In Table 6, we present the experiment setup of our method and RULSTM. Both RULTM and our model (Ours) use various supervisory signals for training, yet our model only needs RGB frames for inference and is end-to-end trainable. Ours+Obj model does require more training signals in comparison to RULSTM, yet it does not need optical flow for two-stream architecture. We have to point out that, from practical prospective, we care more about the data modality during testing time. Therefore, using more supervisory signals for training does not compromise the contribution of our method. Moreover, our method also address the challenging problem of motor attention prediction and interaction hotspots estimation.

\section{J Epic-Kitchens Challenge Leaderboard}

Fig 6 presents a screenshot of the leaderboard from the EPIC-Kitchens Action Anticipation Challenge (https://epic-kitchens.github.io/). ${ }^{5}$ The screenshot was acquired on the last day of supplementary material deadline. To date, our proposed method outperforms all published results by a large margin. Several very recent unpublished work (user id: "action_banks", "reza_zlf", "hepic", "prefact", "root" in Fig. 6) also attempted at the EPIC-Kitchens Action Anticipation Challenge. The only work that outperforms our method is "action_banks". Although "action_banks" slightly outperforms our method for action prediction, their results are worse than our method in terms of the verb and noun prediction.

\section{K Experiment on Gaze Fixation Model}

In this section, we present additional results on using gaze as attention distribution for visual anticipation. We follow [31] to replace motor attention and hotspots modules with a gaze module. We denote the resulting model as Gaze Only model. The experiments are conducted on EGTEA dataset, as gaze is not available on EPIC-Kitchens dataset. Gaze Only model improves the I3D-Res50 baseline by a notable margin. However, it lags behind our full model. This is

\footnotetext{
${ }^{5}$ Retrieved at March 13th, 2020.
} 
because our model explicitly reasons about the future representation by making motor attention a first class player.

\section{Additional Qualitative Results}

Finally, we provide additional qualitative results. We included a video demo of our results as part of our supplementary materials. In this document, we also present more samples of predicted motor attention, interaction hotspots, and action labels in Fig 5. The figure follows the same format as Fig. 3 in the submission. These results further show that our proposed motor attention module has the remarkable ability of "imagining" possible hand movements even without the presence of hands in the observed video segments. Another interesting observation is that the predicted distribution of interaction hostpots can be sparse in certain circumstances (e.g., "Open Fridge" or "Take Condiment"). This is because of the stochastic patterns of human-object interaction: There might be multiple valid contact regions for interaction, especially when the future active object has a relatively large scale. This again shows the necessity of the stochastic units in our proposed method.

As discussed in our submission, the occlusion and absence of active objects might make the anticipation problem extremely challenging even for humans. The failure cases in Fig. 5 also suggest that the anticipation model can be biased by on-going action. This is because current FPV datasets (especially EPIC-Kitchens) segment a continuous action into several same atomic actions to ensure all action segments have similar temporal dimension. For instance, A video clip of "cutting onions" for 20 seconds is segmented into 7 or 8 shorter clips all having the same "cutting onions" label. This increases the transition probability of staying in current action state, and thereby biases the model. Therefore, the ability of predicting when exactly the action will end is important for more accurate action prediction model. This task is also related to the action localization problem in the literature [19]. 


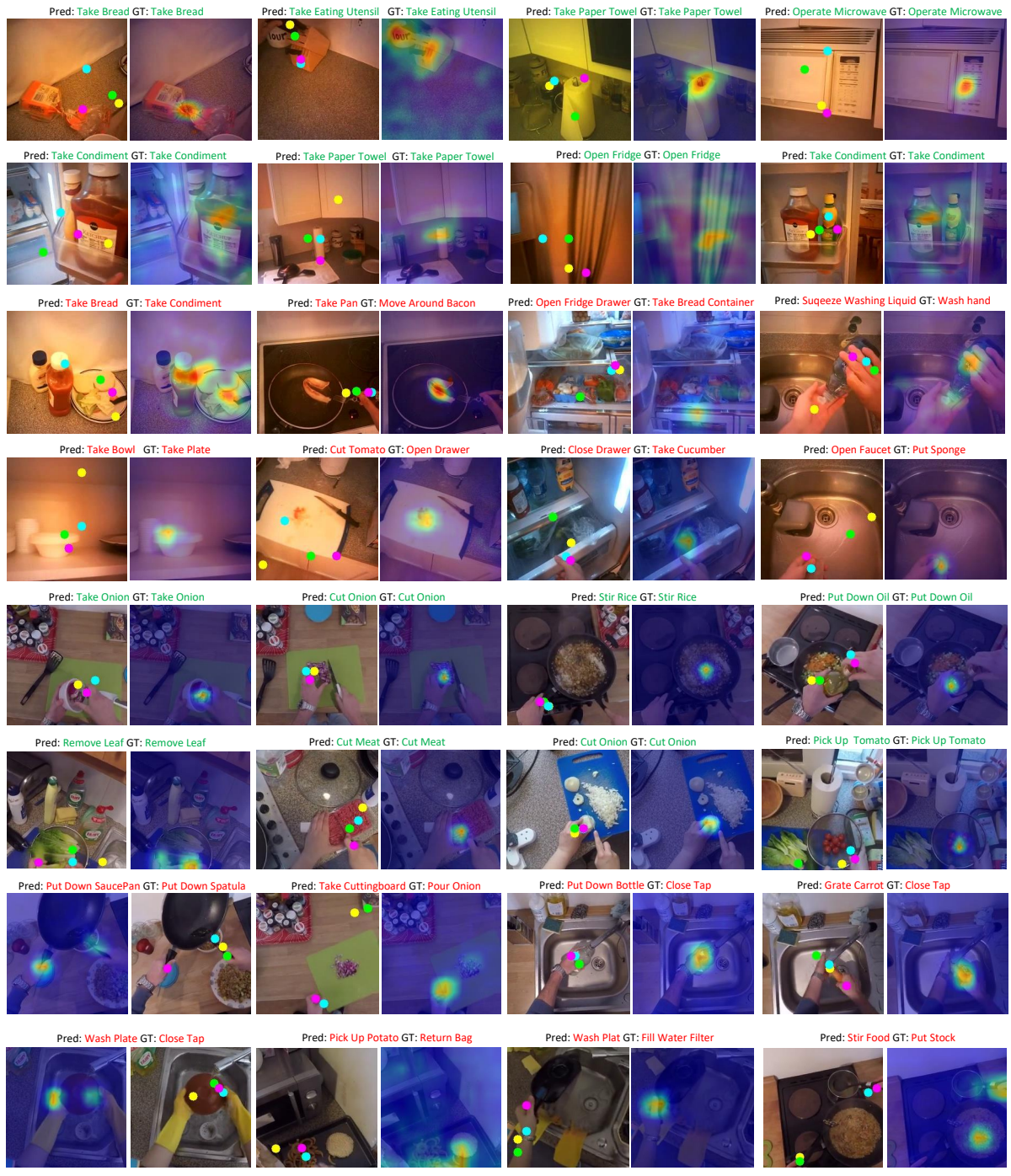

Fig. 5. Additional visualization of predicted motor attention (left), interaction hotspots (right), and action labels (top) from the EGTEA (row 1-4) and EPIC-Kitchens (row 5-8). Both successful cases (green label) and failure cases (red label) are presented. Future hands position are downsampled by a temporal factor of 8 , and forecasted to the last observable frame in the order of yellow, green, cyan, and magenta. 


\begin{tabular}{|c|c|c|c|c|c|c|c|c|c|c|c|c|c|c|c|c|c|c|c|}
\hline \multirow{3}{*}{ \# } & \multirow{3}{*}{ User } & \multirow{3}{*}{ Entries } & \multirow{3}{*}{$\begin{array}{l}\text { Date of last } \\
\text { Entry }\end{array}$} & \multicolumn{6}{|c|}{ Seen Kitchens (S1) } & \multirow{3}{*}{ 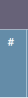 } & \multirow{3}{*}{ User } & \multirow{3}{*}{ Entries } & \multirow{3}{*}{$\begin{array}{l}\text { Date of Last } \\
\text { Entry }\end{array}$} & \multicolumn{6}{|c|}{ Unseen Kitchens (S2) } \\
\hline & & & & \multicolumn{3}{|c|}{ Top-1 Accuracy (\%) } & \multicolumn{3}{|c|}{ Top-5 Accuracy (\%) } & & & & & \multicolumn{3}{|c|}{ Top-1 Accuracy (\%) } & \multicolumn{3}{|c|}{ Top-5 Accuracy (\%) } \\
\hline & & & & Verb $\boldsymbol{\Delta}$ & Noun $\boldsymbol{\Delta}$ & Action & Verb $\boldsymbol{\Lambda}$ & Noun $\boldsymbol{A}$ & Action & & & & & Verb $\Delta$ & Noun & Action & Verb $\boldsymbol{\Delta}$ & Noun $\mathbf{\Delta}$ & Action \\
\hline 1 & action_banks & 15 & $03 / 02 / 20$ & $\begin{array}{l}31.35 \\
\text { (5) }\end{array}$ & $\begin{array}{l}22.64 \\
\text { (6) } \\
\end{array}$ & $\begin{array}{l}16.54 \\
\text { (1) }\end{array}$ & $\begin{array}{l}75.15 \\
(10) \\
\end{array}$ & $\begin{array}{l}47.16 \\
\text { (6) }\end{array}$ & $\begin{array}{l}36.36 \\
(1) \\
\end{array}$ & 1 & action_t & 15 & $03 / 02 / 20$ & $\begin{array}{l}27.48 \\
\text { (3) }\end{array}$ & $\begin{array}{l}16.63 \\
\text { (2) }\end{array}$ & $\begin{array}{l}10.00 \\
(1) \\
\end{array}$ & $\begin{array}{l}66.81 \\
(11) \\
\end{array}$ & $\begin{array}{l}32.84 \\
(6)\end{array}$ & $\begin{array}{l}23.39 \\
\text { (2) }\end{array}$ \\
\hline 2 & aptx48691m & 20 & $11 / 12 / 19$ & $\uparrow_{(1)}^{36.25}$ & $\prod_{(3)}^{23.83}$ & $\downarrow_{(2)}^{15.42}$ & $\begin{array}{l}79.15 \\
\text { (3) } \\
\end{array}$ & $\begin{array}{l}51.98 \\
(3) \\
\end{array}$ & $\begin{array}{l}34.29 \\
\text { (3) }\end{array}$ & 2 & apt $x 4$ & 20 & 19 & $\uparrow_{(2)}^{29.87}$ & $\uparrow_{(1)}^{16.80}$ & $\downarrow_{(2)}^{9.94}$ & $\begin{array}{l}71.77 \\
\text { (2) } \\
\end{array}$ & $\begin{array}{l}38.96 \\
\text { (2) }\end{array}$ & $\begin{array}{l}23.69 \\
\text { (1) }\end{array}$ \\
\hline 3 & antoninofurnari & 1 & 07/19/19 & $\begin{array}{l}31.13 \\
\text { (6) }\end{array}$ & $\begin{array}{l}22.93 \\
(4)\end{array}$ & $\begin{array}{l}15.25 \\
\text { (3) }\end{array}$ & $\begin{array}{l}78.03 \\
(6)\end{array}$ & $\begin{array}{l}51.05 \\
\text { (4) }\end{array}$ & $\begin{array}{l}35.13 \\
\text { (2) }\end{array}$ & 3 & antoninofurnari & 1 & $07 / 19 / 19$ & $\begin{array}{l}26.63 \\
\text { (6) }\end{array}$ & $\begin{array}{l}15.47 \\
\text { (4) }\end{array}$ & $\begin{array}{l}9.12 \\
\text { (3) }\end{array}$ & $\begin{array}{l}68.11 \\
(9)\end{array}$ & $\begin{array}{l}35.27 \\
\text { (4) }\end{array}$ & $\begin{array}{l}21.88 \\
\text { (3) }\end{array}$ \\
\hline 4 & root & 4 & $01 / 09 / 20$ & $\begin{array}{l}33.52 \\
\text { (3) }\end{array}$ & $\begin{array}{l}22.88 \\
(5)\end{array}$ & $\begin{array}{l}14.56 \\
(4)\end{array}$ & $\begin{array}{l}79.17 \\
\text { (2) }\end{array}$ & $\begin{array}{l}50.83 \\
(5)\end{array}$ & $\begin{array}{l}33.83 \\
\text { (4) }\end{array}$ & 4 & root & 4 & $01 / 09 / 20$ & $\begin{array}{l}27.42 \\
\text { (4) }\end{array}$ & $\begin{array}{l}15.30 \\
\text { (5) }\end{array}$ & $\begin{array}{l}8.02 \\
(4)\end{array}$ & $\begin{array}{l}69.14 \\
\text { (6) }\end{array}$ & $\begin{array}{l}35.06 \\
\text { (5) }\end{array}$ & $\begin{array}{l}20.52 \\
\text { (4) }\end{array}$ \\
\hline 5 & hepic & 19 & $11 / 28 / 19$ & $\begin{array}{l}30.76 \\
\text { (7) }\end{array}$ & $\begin{array}{l}17.40 \\
\text { (7) }\end{array}$ & $\begin{array}{l}8.98 \\
(5)\end{array}$ & $\begin{array}{l}77.25 \\
\text { (7) }\end{array}$ & $\begin{array}{l}42.70 \\
(7)\end{array}$ & $\begin{array}{l}24.15 \\
\text { (6) }\end{array}$ & 5 & prefact & 13 & $11 / 12 / 19$ & $\begin{array}{l}23.22 \\
(10)\end{array}$ & $\begin{array}{l}16.18 \\
\text { (3) }\end{array}$ & $\begin{array}{l}4.81 \\
\text { (5) }\end{array}$ & $\begin{array}{l}71.01 \\
\text { (3) }\end{array}$ & $\begin{array}{l}39.23 \\
\text { (1) }\end{array}$ & $\begin{array}{l}14.27 \\
\text { (5) }\end{array}$ \\
\hline 6 & reza_zlf & 3 & 10/28/19 & $\begin{array}{l}29.45 \\
(10)\end{array}$ & $\begin{array}{l}24.78 \\
\text { (2) }\end{array}$ & $\begin{array}{l}8.30 \\
\text { (6) }\end{array}$ & $\begin{array}{l}79.50 \\
\text { (1) }\end{array}$ & $\begin{array}{l}53.16 \\
\text { (2) }\end{array}$ & $\begin{array}{l}25.55 \\
\text { (5) }\end{array}$ & 6 & hepic & 19 & $11 / 28 / 19$ & $\begin{array}{l}27.01 \\
\text { (5) }\end{array}$ & $\begin{array}{l}9.76 \\
\text { (8) }\end{array}$ & $\begin{array}{l}4.64 \\
(6)\end{array}$ & $\begin{array}{l}69.48 \\
(4)\end{array}$ & $\begin{array}{l}27.55 \\
\text { (9) }\end{array}$ & $\begin{array}{l}14.27 \\
\text { (5) }\end{array}$ \\
\hline 7 & prefact & 13 & $11 / 12 / 19$ & $\begin{array}{l}26.32 \\
\text { (11) }\end{array}$ & $\begin{array}{l}25.60 \\
\text { (1) }\end{array}$ & $\begin{array}{l}7.44 \\
\text { (7) }\end{array}$ & $\begin{array}{l}79.10 \\
\text { (4) }\end{array}$ & $\begin{array}{l}53.18 \\
\text { (1) }\end{array}$ & $\begin{array}{l}23.10 \\
\text { (7) }\end{array}$ & 7 & ri & 1 & 9/19 & $\begin{array}{l}32.37 \\
\text { (1) }\end{array}$ & $\begin{array}{l}9.66 \\
\text { (9) }\end{array}$ & $\begin{array}{l}3.52 \\
(7)\end{array}$ & $\begin{array}{l}73.51 \\
\text { (1) }\end{array}$ & $\begin{array}{l}30.83 \\
(7)\end{array}$ & $\begin{array}{l}12.67 \\
\text { (7) }\end{array}$ \\
\hline 8 & EPIC_TSN_RGB & 1 & $09 / 05 / 18$ & $\begin{array}{l}31.81 \\
(4)\end{array}$ & $\begin{array}{l}16.22 \\
(8)\end{array}$ & $\begin{array}{l}6.00 \\
(8)\end{array}$ & $\begin{array}{l}76.56 \\
(8)\end{array}$ & $\begin{array}{l}42.15 \\
\text { (8) }\end{array}$ & $\begin{array}{l}18.21 \\
\text { (8) }\end{array}$ & 8 & reza_zlf & 3 & 19 & $\begin{array}{l}22.29 \\
(12)\end{array}$ & $\begin{array}{l}14.10 \\
(6)\end{array}$ & $\begin{array}{l}3.18 \\
\text { (8) }\end{array}$ & $\begin{array}{l}69.38 \\
(5)\end{array}$ & $\begin{array}{l}35.47 \\
\text { (3) }\end{array}$ & $\begin{array}{l}12.91 \\
\text { (6) }\end{array}$ \\
\hline 9 & yassersou & 1 & 08/19/19 & $\begin{array}{l}34.94 \\
\text { (2) }\end{array}$ & $\begin{array}{l}13.06 \\
(10)\end{array}$ & $\begin{array}{l}5.23 \\
(9)\end{array}$ & $\begin{array}{l}79.07 \\
(5)\end{array}$ & $\begin{array}{l}37.00 \\
(10)\end{array}$ & $\begin{array}{l}16.27 \\
\text { (9) }\end{array}$ & 9 & B & 1 & 18 & $\begin{array}{l}25.30 \\
\text { (9) }\end{array}$ & $\begin{array}{l}10.41 \\
\text { (7) }\end{array}$ & $\begin{array}{l}2.39 \\
(9)\end{array}$ & $\begin{array}{l}68.32 \\
\text { (7) }\end{array}$ & $\begin{array}{l}29.50 \\
\text { (8) }\end{array}$ & $\begin{array}{l}9.63 \\
(8)\end{array}$ \\
\hline 10 & EPIC_TSN_Fusion & 1 & 09/05/18 & $\begin{array}{l}30.66 \\
(8)\end{array}$ & $\begin{array}{l}14.86 \\
(9)\end{array}$ & $\begin{array}{l}4.62 \\
\text { (10) }\end{array}$ & $\begin{array}{l}75.32 \\
\text { (9) }\end{array}$ & $\begin{array}{l}40.11 \\
\text { (9) }\end{array}$ & $\begin{array}{l}16.01 \\
(10)\end{array}$ & 10 & low & 1 & i/18 & $\begin{array}{l}25.61 \\
\text { (7) }\end{array}$ & $\begin{array}{l}8.40 \\
(10)\end{array}$ & $\begin{array}{l}1.78 \\
(10)\end{array}$ & $\begin{array}{l}67.57 \\
(10)\end{array}$ & $\begin{array}{l}24.62 \\
(11)\end{array}$ & $\begin{array}{l}8.19 \\
(10)\end{array}$ \\
\hline 11 & EPIC_TSN_Flow & 1 & 09/05/18 & $\begin{array}{l}29.64 \\
(9)\end{array}$ & $\begin{array}{l}10.30 \\
(11)\end{array}$ & $\begin{array}{l}2.93 \\
(11)\end{array}$ & $\begin{array}{l}73.70 \\
(11)\end{array}$ & $\begin{array}{l}30.09 \\
(11)\end{array}$ & $\begin{array}{l}10.92 \\
(11)\end{array}$ & 11 & Fusion & 1 & 18 & $\begin{array}{l}25.37 \\
(8)\end{array}$ & $\begin{array}{l}9.76 \\
(8)\end{array}$ & $\begin{array}{l}1.74 \\
(11)\end{array}$ & $\begin{array}{l}68.25 \\
(8)\end{array}$ & $\begin{array}{l}27.24 \\
(10)\end{array}$ & $\begin{array}{l}9.05 \\
(9)\end{array}$ \\
\hline 12 & jianjiangkcl & 13 & 08/21/19 & $\begin{array}{l}21.55 \\
(13)\end{array}$ & $\begin{array}{l}5.52 \\
(13)\end{array}$ & $\begin{array}{l}2.04 \\
(12)\end{array}$ & $\begin{array}{l}68.81 \\
(13)\end{array}$ & $\begin{array}{l}19.17 \\
(13)\end{array}$ & $\begin{array}{l}5.85 \\
(12)\end{array}$ & 12 & net & 2 & 18 & $\begin{array}{l}22.36 \\
(11)\end{array}$ & $\begin{array}{l}6.59 \\
(11)\end{array}$ & $\begin{array}{l}0.89 \\
(12)\end{array}$ & $\begin{array}{l}63.43 \\
(12)\end{array}$ & $\begin{array}{l}19.73 \\
(12)\end{array}$ & $\begin{array}{l}3.72 \\
(11)\end{array}$ \\
\hline 13 & masterchef & 2 & 09/06/18 & $\begin{array}{l}25.30 \\
(12)\end{array}$ & $\begin{array}{l}7.78 \\
(12)\end{array}$ & $\begin{array}{l}1.58 \\
(13)\end{array}$ & $\begin{array}{l}69.98 \\
(12)\end{array}$ & $\begin{array}{l}22.07 \\
(12)\end{array}$ & $\begin{array}{l}5.85 \\
\text { (12) }\end{array}$ & 13 & jianjiangkcl & ${ }^{13}$ & $08 / 21 / 19$ & $\begin{array}{l}16.08 \\
(13)\end{array}$ & $\begin{array}{l}3.28 \\
(12)\end{array}$ & $\begin{array}{l}0.68 \\
(13)\end{array}$ & $\begin{array}{l}59.88 \\
(13)\end{array}$ & $\begin{array}{l}13.28 \\
(13)\end{array}$ & $\begin{array}{l}2.77 \\
(12)\end{array}$ \\
\hline
\end{tabular}

Fig. 6. Screenshot from Epic-Kitchens Anticipation Challenge. The user name of our proposed method is "aptx4869lm". The current rank1 team "action_banks" is unpublished work, and lags behind of our method for both verb and noun prediction on both sets. Note that user "antonionfurnari" refers to RULSTM in our main submission. They further improved the results reported in their paper. 


\begin{tabular}{|c|c|c|c|c|c|c|}
\hline ID & Branch & Type & $\begin{array}{c}\text { Kernel Size } \\
\text { THW,(C) }\end{array}$ & $\begin{array}{l}\text { Stride } \\
\text { THW }\end{array}$ & $\begin{array}{c}\text { Output Size } \\
\text { THWC }\end{array}$ & Comments (Loss) \\
\hline 1 & & Conv3D & $5 \times 7 \times 7,64$ & $2 \times 2 \times 2$ & $16 \times 112 \times 112 \times 64$ & \\
\hline 2 & & MaxPool1 & $2 \times 3 \times 3$ & $2 \times 2 \times 2$ & $8 \times 56 \times 56 \times 64$ & \\
\hline 3 & & $\begin{array}{l}\text { Layer1 } \\
\text { Bottleneck 0-2 }\end{array}$ & $\begin{array}{l}3 \times 1 \times 1,64 \\
1 \times 3 \times 3,64 \text { (3 times) } \\
1 \times 1 \times 1,256\end{array}$ & $\begin{array}{l}1 \mathrm{x} 1 \mathrm{x} 1 \\
1 \mathrm{x} 1 \mathrm{x} 1 \text { (3 times) } \\
1 \mathrm{x} 1 \mathrm{x} 1\end{array}$ & $8 \times 56 \times 56 \times 256$ & \\
\hline 4 & & MaxPool2 & $2 \times 1 \times 1$ & $2 \times 1 \times 1$ & $4 \times 56 \times 56 \times 256$ & $\begin{array}{c}\text { Addition Pooling } \\
\text { Reduce Memory Usage }\end{array}$ \\
\hline 5 & & $\begin{array}{c}\text { Layer2 } \\
\text { Bottleneck } 0\end{array}$ & $\begin{array}{l}3 \times 1 \times 1,128 \\
1 \times 3 \times 3,128 \\
1 \times 1 \times 1,512\end{array}$ & $\begin{array}{l}1 \times 1 \times 1 \\
1 \times 2 \times 2 \\
1 \times 1 \times 1\end{array}$ & & \\
\hline 6 & $\begin{array}{l}\text { Backbone } \\
\text { (shared) }\end{array}$ & $\begin{array}{l}\text { Layer2 } \\
\text { Bottleneck 1-3 }\end{array}$ & \begin{tabular}{|l|}
$3 \times 1 \times 1,128$ \\
$1 \times 3 \times 3,128$ (3 times) \\
$1 \times 1 \times 1,512$ \\
\end{tabular} & $\begin{array}{l}1 \times 1 \times 1 \\
1 \times 2 \times 2 \text { (3 times) } \\
1 \times 1 \times 1\end{array}$ & $4 \times 28 \times 28 \times 512$ & \\
\hline 7 & & $\begin{array}{c}\text { Layer3 } \\
\text { Bottleneck } 0\end{array}$ & $\begin{array}{l}3 \times 1 \times 1,256 \\
1 \times 3 \times 3,256 \\
1 \times 1 \times 1,1024 \\
\end{array}$ & $\begin{array}{l}1 \mathrm{x} 1 \mathrm{x} 1 \\
1 \mathrm{x} 2 \mathrm{x} 2 \\
1 \mathrm{x} 1 \mathrm{x} 1\end{array}$ & & \\
\hline 8 & & $\begin{array}{c}\text { Layer3 } \\
\text { Bottleneck 1-5 }\end{array}$ & $\begin{array}{l}3 \times 1 \times 1,256 \\
1 \times 3 \times 3,256 \quad(5 \text { times }) \\
1 \times 1 \times 1,1024\end{array}$ & $\begin{array}{l}1 \mathrm{x} 1 \mathrm{x} 1 \\
1 \mathrm{x} 1 \mathrm{x} 1 \text { (5 times) } \\
1 \mathrm{x} 1 \mathrm{x} 1\end{array}$ & $4 \times 14 \times 14 \times 1024$ & \\
\hline 9 & & $\begin{array}{c}\text { Layer4 } \\
\text { Bottleneck } 0\end{array}$ & $\begin{array}{l}3 \times 1 \times 1,128 \\
1 \times 3 \times 3,128 \\
1 \times 1 \times 1,512\end{array}$ & $\begin{array}{l}1 \mathrm{x} 1 \mathrm{x} 1 \\
1 \mathrm{x} 2 \mathrm{x} 2 \\
1 \mathrm{x} 1 \mathrm{x} 1\end{array}$ & & \\
\hline 10 & & $\begin{array}{c}\text { Layer4 } \\
\text { Bottleneck 1-2 }\end{array}$ & \begin{tabular}{|l|}
$\mathrm{x} 1 \mathrm{x} 1,128$ \\
$1 \times 3 \times 3,128$ (2 times) \\
$1 \times 1 \times 1,512$
\end{tabular} & $\begin{array}{l}1 \times 1 \times 1 \\
1 \times 2 \times 2 \text { (2 times) } \\
1 \times 1 \times 1\end{array}$ & $4 \times 7 \times 7 \times 2048$ & \\
\hline 11 & \multirow{6}{*}{$\begin{array}{c}\text { Motor } \\
\text { Attention } \\
\text { Module }\end{array}$} & $\begin{array}{c}\text { Conv3d } 1 \\
\text { (on Layer } 2 \text { feature) }\end{array}$ & $1 \times 3 \times 3,128$ & $1 \times 1 \times 1$ & $4 \times 28 \times 28 \times 128$ & \\
\hline 12 & & Conv3d 2 & $1 \times 3 \times 3,1$ & $1 \times 1 \times 1$ & $4 \times 28 \times 28 \times 1$ & KLD Loss \\
\hline 13 & & Maxpool 1 & $1 \times 2 \times 2$ & $1 \times 2 \times 2$ & $4 \times 14 \times 14 \times 1$ & Guiding Interaction Hotspots \\
\hline 14 & & $\begin{array}{c}\text { Gumbel Softmax } 1 \\
\text { (Sampling) }\end{array}$ & & & $4 \times 14 \times 14 \times 1$ & Sampling Motor Attention \\
\hline 15 & & Maxpool 2 & $1 \times 4 \times 4$ & $1 \times 4 \times 4$ & $4 \times 7 \times 7 \times 1$ & Guiding Action Anticipation \\
\hline 16 & & $\begin{array}{c}\text { Gumbel Softmax } 2 \\
\text { (Sampling) }\end{array}$ & & & $4 \times 7 \times 7 \times 1$ & Sampling Motor Attention \\
\hline 17 & \multirow{5}{*}{$\begin{array}{c}\text { Interaction } \\
\text { Hotspots } \\
\text { Module }\end{array}$} & Weighted Pooling & & & $4 \times 14 \times 14 \times 256$ & With Sampled Motor Attention \\
\hline 18 & & \begin{tabular}{|c|} 
Conv3d 1 \\
(on Layer 3 Feature)
\end{tabular} & $1 \times 3 \times 3,256$ & $1 \times 1 \times 1$ & $4 \times 14 \times 14 \times 256$ & \\
\hline 19 & & Conv3d 2 & $1 \times 3 \times 3,1$ & $1 \mathrm{x} 1 \mathrm{x} 1$ & $4 \times 14 \times 14 \times 1$ & KLD Loss \\
\hline 20 & & Maxpool 1 & $1 \times 2 \times 2$ & $1 \times 2 \times 2$ & $4 \times 7 \times 7 \times 1$ & Guiding Action Anticipation \\
\hline 21 & & $\begin{array}{l}\text { Gumbel Softmax } \\
\text { (Sampling) }\end{array}$ & & & $4 \times 7 \times 7 \times 1$ & Sampling Interaction Hotspots \\
\hline 22 & \multirow{3}{*}{$\begin{array}{c}\text { Action } \\
\text { Anticipation } \\
\text { Module }\end{array}$} & $\begin{array}{c}\text { Weighted } \\
\text { Avg Pool } \\
\text { (on Final Feature) }\end{array}$ & $4 \times 7 \times 7$ & $4 \times 7 \times 7$ & $1 \times 1 \times 1 \times 1024$ & $\begin{array}{l}\text { With Sampled Motor Attention } \\
\text { and Interaction Hotspots }\end{array}$ \\
\hline 23 & & Fully Connected & & & $1 \mathrm{x} 1 \mathrm{x} 1 \mathrm{xN}$ & \\
\hline 24 & & Softmax & & & $1 \mathrm{x} 1 \mathrm{x} 1 \mathrm{xN}$ & $\begin{array}{l}\text { Cross Entropy Loss } \\
\text { (Action Anticipation) }\end{array}$ \\
\hline
\end{tabular}

Table 8. Network architecture of our proposed model. We omit the residual connection in backbone ResNet-50 for simplification. 